\title{
Arquitectura ceremonial inca en las tierras altas del noroeste argentino: caracterización de los ushnus de Cueva Inca Viejo y Abra de Minas, puna de Salta
}

Gabriel E. J. López y Federico I. Coloca

\section{OpenEdition}

\section{Journals}

Edición electrónica

URL: http://journals.openedition.org/bifea/10707

DOI: 10.4000/bifea.10707

ISSN: 2076-5827

\section{Editor}

Institut Français d'Études Andines

\section{Edición impresa}

Fecha de publicación: 1 agosto 2019

Paginación: 229-237

ISSN: 0303-7495

\section{Referencia electrónica}

Gabriel E. J. López y Federico I. Coloca, « Arquitectura ceremonial inca en las tierras altas del noroeste argentino: caracterización de los ushnus de Cueva Inca Viejo y Abra de Minas, puna de Salta », Bulletin de l'Institut français d'études andines [En línea], 48 (2) | 2019, Publicado el 08 agosto 2019, consultado el 29 noviembre 2020. URL : http://journals.openedition.org/bifea/10707 ; DOI : https://doi.org/10.4000/ bifea. 10707

Les contenus du Bulletin de l'Institut français d'études andines sont mis à disposition selon les termes de la licence Creative Commons Attribution - Pas d'Utilisation Commerciale - Pas de Modification 4.0 International. 


\title{
Arquitectura ceremonial inca en las tierras altas del noroeste argentino: caracterización de los ushnus de Cueva Inca Viejo y Abra de Minas, puna de Salta
}

\author{
Gabriel E. J. López* \\ Federico I. Coloca**
}

\section{INTRODUCCIÓN}

Este trabajo presenta nuevas evidencias para comprender los aspectos simbólicorituales en la expansión inca al sur del Tawantinsuyu, específicamente en las tierras altas del noroeste argentino. Se ha destacado el importante rol que el Imperio inca asignó al control ideológico y simbólico de las poblaciones locales, a partir de la propiciación de prácticas rituales y materialidad asociada (e.g. D’Altroy, 2015). Concretamente, los incas impusieron su ideología a través de la realización de ceremonias y fiestas de hospitalidad, manipulación de objetos simbólicos y arquitectura imperial, entre otros aspectos (DeMarrais et al., 1996; Williams et al., 2005). Al respecto, cabe señalar la relevancia de la arquitectura ceremonial inca como parte de la apropiación simbólica de los espacios dominados a lo largo del Tawantinsuyu. En especial, se hace referencia a construcciones denominadas ushnus, caracterizadas por una amplia diversidad de formas y tamaños en las distintas regiones del Imperio (Monteverde Sotil, 2011). Debe considerarse que el término ushnu remite a espacios de uso ceremonial para la realización de libaciones y ofrendas (Zuidema, 2014). En consecuencia, la arquitectura ceremonial estaría asociada a una función libatoria y ofrendatoria del ushnu, que

* CONICET. Instituto de Arqueología, FFyL, UBA (25 de mayo 217, $3^{\circ}$ piso, Ciudad de Buenos Aires, Código postal: 1002,Argentina).E-mail: gabelope@yahoo.com

** CONICET. Instituto de Arqueología,FFyL, UBA. E-mail: fedeigco@hotmail.com 
conectaría el mundo terrenal con el mundo subterráneo a través de conductos para la circulación de los líquidos (Zuidema, 2014). Más allá de la discusión acerca de sus distintas definiciones, los ushnus se suelen reconocer arqueológicamente a partir de características arquitectónicas y su asociación con aspectos simbólicorituales (Farrington, 1999; Hyslop, 1990; McEwan, 2014; Meddens, 2014; Raffino, 1981; entre otros). Entre la variabilidad arquitectónica detectada se mencionan plataformas rectangulares, cuadrangulares o trapezoidales, con uno o más niveles y, en algunos casos, con escalinatas o rampas (Monteverde Sotil, 2011). En la región de estudio, el noroeste argentino, estas características variables se observan en distintos sitios incas, tanto en tierras altas como en valles y quebradas (e.g. Acuto et al., 2012; Farrington, 1999; Leibowicz et al., 2016; Lynch et al., 2010; Moralejo, 2011; Palma et al., 2006; Raffino, 1981; Williams, 2000).

A partir de las consideraciones previas, este trabajo analiza y discute brevemente dos nuevos casos de arquitectura ceremonial que pueden ser definidos como ushnus. Específicamente, la evidencia presentada proviene de los sitios Cueva Inca Viejo y Abra de Minas, localizados en la cuenca de Ratones, puna de Salta, noroeste argentino (fig. 1). Estos sitios formaron parte de un paisaje mineroritual construido por el Tawantinsuyu en el área de Ratones (véase próxima sección). En el mundo andino, la asociación entre minería y ritualidad ha sido destacada en distintos casos (e.g. Platt et al., eds., 2006). A su vez, el vínculo entre arquitectura ceremonial y explotación minera también ha sido mencionado en otros espacios de las tierras altas surandinas (e.g. Soto Rodríguez \& Salazar Sutil, 2016). En consecuencia, esta nueva evidencia aporta a la comprensión del control ideológico y la apropiación efectiva y simbólica de un espacio productivo-minero por parte del Imperio inca.

\section{EL USHNU DE CUEVA INCA VIEJO}

Cueva Inca Viejo se ubica en el sector oeste del cerro homónimo a una altura de 4312 metros sobre el nivel del mar. Entre las características principales de la cueva se destaca la presencia de una cámara de acceso con diversos paneles de pinturas rupestres y galerías secundarias abiertas por mineros prehispánicos ${ }^{1}$. En este sentido, debe señalarse que el Cerro Inca Viejo, donde se ubica la cueva, constituye una fuente del mineral turquesa. Por esta razón, Cueva Inca Viejo ha sido un espacio importante para la explotación prehispánica de este mineral, evidenciada a partir de la presencia de rocas con turquesa en contextos estratigráficos y artefactos asociados con esta actividad, en especial martillos líticos y cueros para su enmangue (López et al., 2018).

En las excavaciones realizadas en el interior de la cueva (16 sondeos de 0,5 x 0,5 m y una cuadrícula de $1 \times 1 \mathrm{~m}$ ) se registraron distintos indicadores relacionados con prácticas rituales, entre ellos, ofrendas de gramíneas con huesos de camélido,

1 Para mayor detalle de la planta del sitio, véase López et al. (2015). 


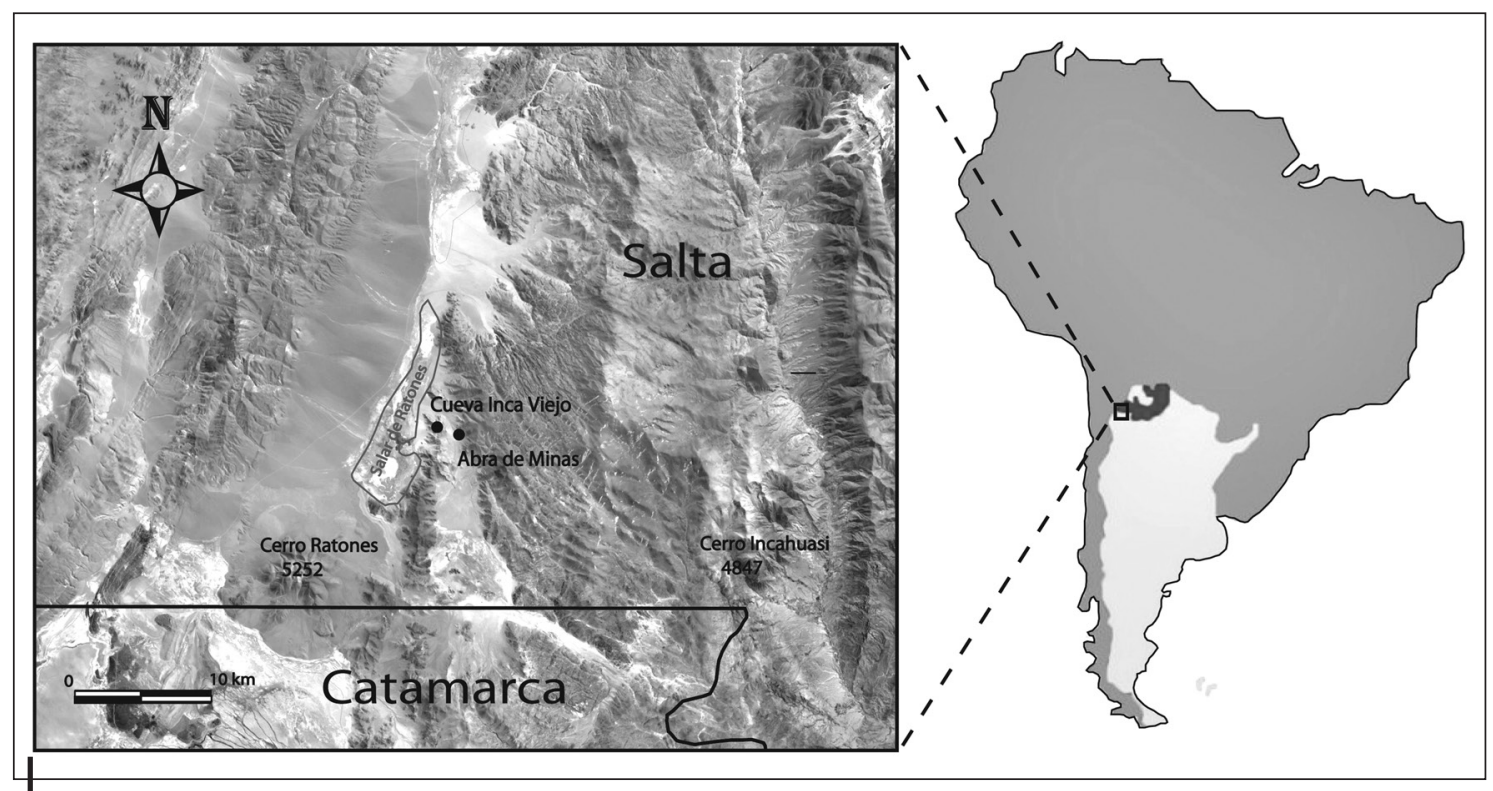

Figura 1 - Ubicación de los sitios Cueva Inca Viejo y Abra de Minas

Elaboración: Federico Coloca

plumas de aves exóticas, cuentas y fragmentos de turquesa y semillas provenientes de plantas alucinógenas como el cebil, originarias de tierras bajas². Sin embargo, el indicador más destacado asociado a este tipo de prácticas está constituido por una estructura ceremonial inca ubicada en el talud de entrada de la cueva (López et al., 2018; López \& Coloca, 2019). Si bien el sitio se viene investigando desde el año 2009, esta construcción no podía ser vista porque se encontraba oculta debajo de rocas de diversos tamaños que cubrían dicho talud, a excepción de un muro superior (fig. 2A). A partir de la remoción de rocas en la campaña de 2017 se comenzaron a descubrir las distintas partes de la estructura, incluyendo una escalera y una plataforma (fig. 2B). Durante la campaña de 2018 se continuó la limpieza de este sector y se distinguieron un muro inferior y un recinto central (figs. 2C y 2D). Estos trabajos permitieron observar la presencia de elementos y técnicas constructivas incaicas en las distintas partes de la estructura. Brevemente, sus características generales son las siguientes: la presencia de un muro doble superior de 2,12 $\mathrm{m}$ de altura con rocas canteadas y argamasa, que obstaculiza la visión de la entrada de la cueva; una plataforma de 1 x 4 m inmediatamente por debajo del muro superior, confeccionada con rocas canteadas y rellena de sedimento; una escalera que sube hacia la cueva, compuesta de 19 escalones elaborados con rocas verticales y relleno de sedimento, y delimitada por muros dobles con canteado y argamasa en ambos costados; y un muro simple inferior

2 Para información más detallada, véase López et al. (2015; 2018). 


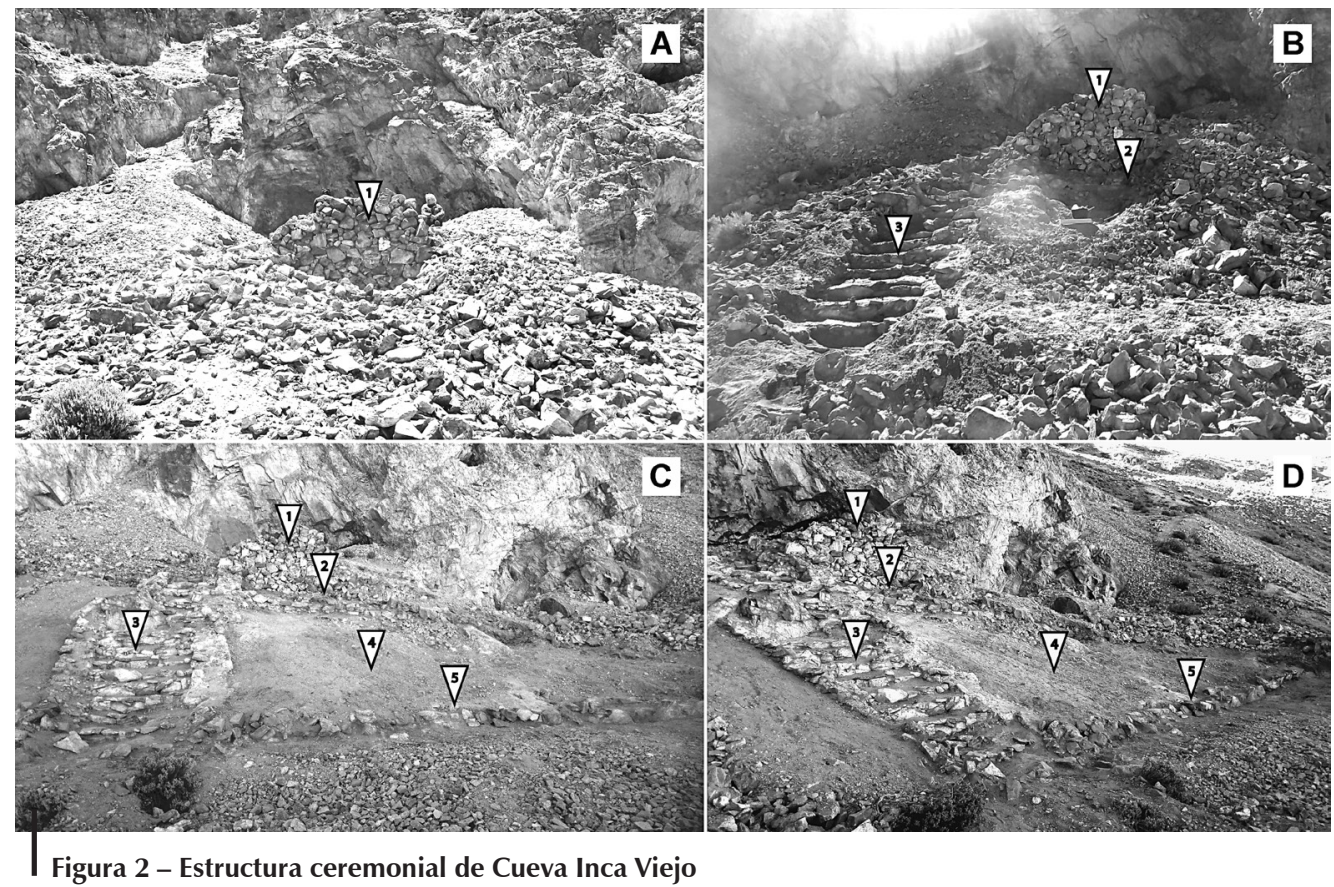

A: Vista inicial con la cobertura de rocas que la ocultaban, B: proceso de descubrimiento, C: vista de frente, D: vista de perfil

1: Muro superior, 2: plataforma rectangular, 3: escalera, 4: recinto central, 5: muro inferior

(C) Gabriel López y Federico Coloca

con rocas canteadas que delimitan la parte baja de la estructura en una extensión de $10 \mathrm{~m}$ aproximadamente. Estas distintas partes encierran un recinto central en pendiente, entre la plataforma y el muro inferior. En relación con la escalera, su morfología y técnica constructiva son similares a las registradas en uno de los cerros aterrazados del sitio inca El Shincal, en los valles de Catamarca, noroeste argentino (Raffino, 1981).

Hasta el momento se realizaron dos sondeos de 0,5 x 0,5 m en la plataforma, los cuales presentaron escasa evidencia arqueológica (e.g. un fragmento de cerámica inca). Sin embargo, en el recinto central de la estructura, durante los trabajos de remoción, se recuperó un monolito de morfología cónica de 43 cm de largo máximo con una cara circular aplanada de $12 \mathrm{~cm}$ de diámetro (López \& Coloca, 2019) (fig. 3A). A su vez, al costado del muro superior se descubrió un segundo monolito de $35,5 \mathrm{~cm}$ de largo máximo con características similares al anterior (fig. 3B). Este tipo de monolitos se ha vinculado a prácticas rituales incaicas para el culto a ancestros notables y deidades, principalmente el Sol (McEwan, 2014; Meddens, 2014; Zuidema, 2014). Específicamente, las fuentes etnohistóricas han destacado el importante rol de monolitos similares en los rituales libatorios desarrollados en el ushnu de la plaza central de Cusco durante el apogeo del 


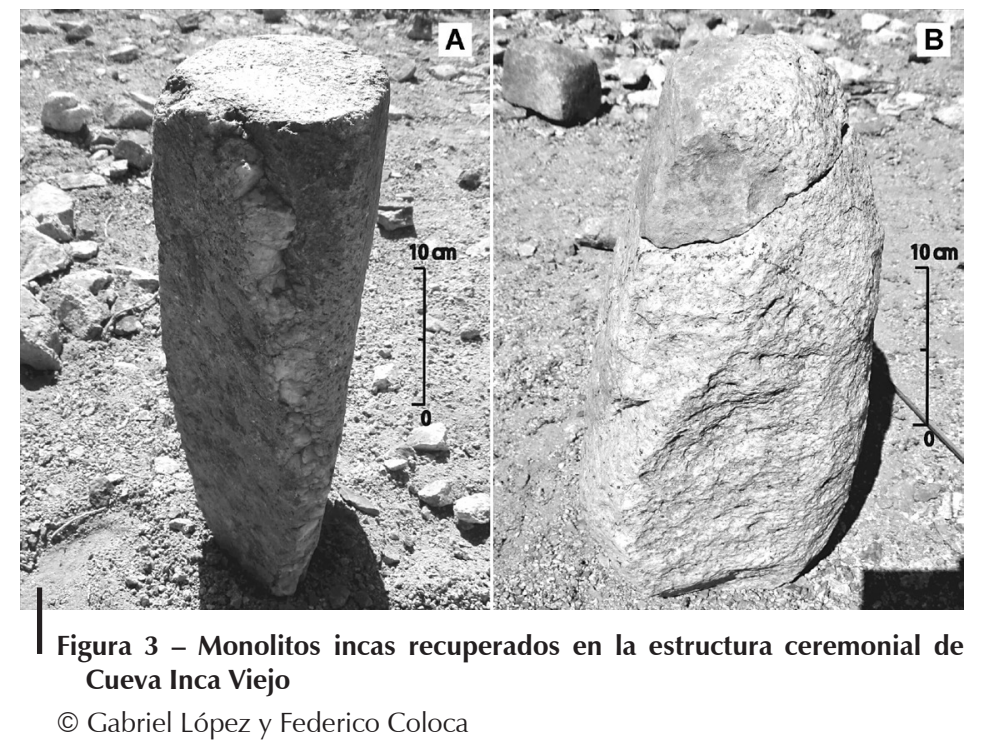

Imperio inca (Meddens, 2014; Zuidema, 2014). A su vez, han sido encontrados en contextos rituales de otros sectores del Tawantinsuyu (Meddens, 2014). La presencia de estos artefactos refuerza la relevancia ceremonial de la estructura y su vínculo con rituales libatorios y ofrendatorios incas, lo cual permite definirla en su totalidad (incluyendo sus distintas partes) como un ushnu. En diversos contextos también se han hallado otros tipos de monolitos que fueron interpretados como gnomones vinculados a prácticas astronómicas incas (Farrington, 1999).

\section{EL USHNU DE ABRA DE MINAS}

Abra de Minas se ubica a 2 km en dirección este de Cueva Inca Viejo y se caracteriza por la gran magnitud constructiva a 4250 metros sobre el nivel del mar ${ }^{3}$. Al respecto, se registran 92 estructuras arquitectónicas, entre las cuales se reconocen formas típicamente incaicas: una kallanka y dos recintos perimetrales compuestos (RPC). La intensa ocupación del Tawantinsuyu en el sitio se documenta también a partir de la alta proporción de cerámica inca (85,9\% del conjunto decorado) (Coloca, 2017). Esta evidencia ha sido interpretada como indicio de celebraciones y fiestas de hospitalidad y comensalismo, considerando la alta representación de aríbalos y platos (Coloca, 2017).

En una vega localizada en el sector noreste de Abra de Minas se ha detectado una plataforma de manufactura inca. Se trata de una estructura de 3,12 x 3,37 m y $0,72 \mathrm{~m}$ de altura máxima cuyos muros presentan rocas canteadas y argamasa. En el año 2019 se realizó una limpieza del sedimento que cubría la superficie de la

3 Para mayor detalle de la planta del sitio, véase Coloca (2017); López \& Coloca (2015). 
plataforma y se descubrió la existencia de un piso compuesto de rocas trabajadas y acomodadas (figs. 4A y 4B). Esta característica constituye otro indicador asociado a técnicas constructivas incas. A su vez, dentro de este espacio se encuentra una división interna marcada por un piso sobreelevado (figs. 4B y 4C). La plataforma se ubica en el centro de la vega, en la pendiente que desciende de los cerros orientales (fig. 4D). A partir de sus atributos arquitectónicos, su posición frente al Cerro Inca Viejo y su ubicación en la pendiente de la vega por donde fluye el agua, esta plataforma puede ser definida como un ushnu (figs. 4C y 4D).

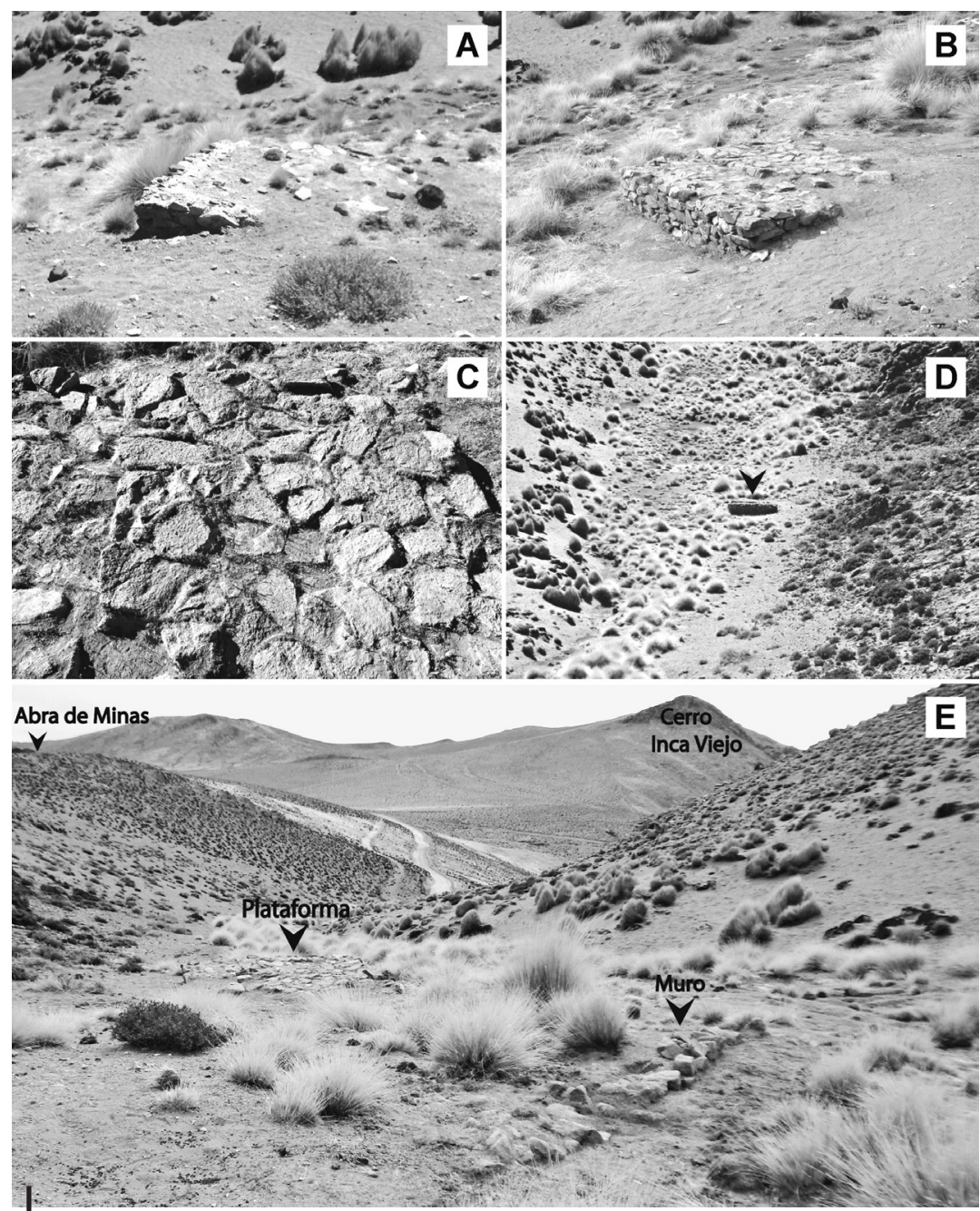

Figura 4 - Plataforma en Abra de Minas

A: Vista original, B: vista general posterior a la limpieza, C: detalle del piso, D: vista de su ubicación en la vega, E: muro por encima de la plataforma

(C) Gabriel López y Federico Coloca 
Asimismo, la presencia de un muro doble de 5,2 m de largo con rocas canteadas, localizado a una distancia máxima de 7,3 $\mathrm{m}$ al este de la plataforma, se relacionaría con el desvío del agua para preservar el ushnu (fig. 4E). En los alrededores de la plataforma se realizaron cuatro sondeos de $0,5 \times 0,5 \mathrm{~m}$, y la evidencia arqueológica fue nula.

\section{CONCLUSIÓN}

A partir del registro presentado en este trabajo se describen dos nuevos ushnus en las tierras altas del noroeste argentino. En el caso de Cueva Inca Viejo se ha descubierto una estructura compleja en la entrada del sitio, con la presencia de monolitos que en otros contextos han sido relacionados con prácticas rituales libatorias y ofrendatorias comúnmente asociadas a la definición de ushnus (Zuidema, 2014). Por su parte, en Abra de Minas se documentó la presencia de una plataforma cuyos elementos constructivos y su ubicación en la pendiente de una vega por la que desciende el flujo de agua constituyen atributos que en otros sitios han permitido caracterizar ushnus.

La complejidad de los elementos que los definen, tales como espacios para libaciones y ofrendas, o como arquitecturas particulares (e.g. plataformas escalonadas), ha sido discutida ampliamente (e.g. Zuidema, 2014). En los casos de Cueva Inca Viejo y Abra de Minas, la información arqueológica reúne dichos aspectos. En consecuencia, el conjunto de los indicadores arquitectónicos, contextuales y artefactuales de ambos casos permite definirlos como ushnus incas. Asimismo, estas estructuras se interpretan como una manifestación concreta de la apropiación efectiva y simbólica de un espacio productivo por parte de los incas. En otras palabras, se plantea aquí que los ushnus constituyeron elementos fundamentales en la dominación ideológico-simbólica del área y en la construcción de un paisaje minero-ritual. En este marco, Cueva Inca Viejo puede ser caracterizada como una huaca minera, es decir, un espacio considerado sagrado donde las prácticas económicas y rituales se entrelazaron.

\section{Referencias citadas}

ACUTO, F. A., TRONCOSO, A. \& FERRARI, A., 2012 - Recognising strategies for conquered territories: a case study from the Inka North Calchaquí Valley. Antiquity, 86 (334): 1141-1154.

COLOCA, F. I., 2017 - Interacciones sociales, prácticas rituales y procesos de cambio durante los Períodos Tardío e Inca (ca. 900-1536 DC) en la cuenca de Ratones, Puna de Salta; Buenos Aires: Universidad de Buenos Aires, Facultad de Filosofía y Letras. Tesis doctoral inédita. 
D'AlTROY, T. N., 2015 - The Incas, xvii + 547 pp.; Chichester, West Sussex: Willey Blackwell.

DeMARRAIS, E., CASTILLO, L. J. \& EARLE, T., 1996 - Ideology, Materialization, and Power Strategies. Current Anthropology, 37 (1):15-31.

FARRINGTON, I., 1999 - El Shincal: un Cusco del Kollasuyu. In: Actas del XII Congreso Nacional de Arqueología Argentina, tomo I (C. Diez Marín, ed.): 53-62; La Plata: Universidad Nacional de La Plata.

HYSLOP, J., 1990 - Inca Settlement Planning, xv + 377 pp.; Austin, TX: University of Texas Press.

LEIBOWICZ, I., MOYANO, R., FERRARI, A., ACUTO, F. \& JACOB, C., 2016 Archaeoastronomy on Inca Sites in the Argentine Northwest. Journal of Skyscape Archaeology, 2 (2): 165-187.

LÓPEZ, G. E. J. \& COLOCA, F. I., 2015 - El sitio Abra de Minas: nuevos aportes para la caracterización de las ocupaciones Tardío/Incas en las tierras altas del noroeste argentino. Bulletin de I'Institut Français d'Études Andines, 44 (1): 141-149.

LÓPEZ, G. E. J. \& COLOCA, F. I., 2019 - Prácticas rituales incas en el Noroeste argentino: hallazgo de un monolito en una estructura ceremonial en Cueva Inca Viejo, Puna de Salta, Argentina. Relaciones de la Sociedad Argentina de Antropología, XLIV (1): 179-186.

LÓPEZ, G. E. J., COLOCA, F. I., ARAYA, S., ORSI, J. P. \& SEGUÍ, S., 2015 - El sitio Cueva Inca Viejo, salar de Ratones, Puna de Salta: Evidencia arqueológica y procesos de interacción macrorregional. Relaciones de la Sociedad Argentina de Antropología, XL (1): 45-71.

LÓPEZ, G. E. J., COLOCA, F. I., ROSENBUSCH, M. \& SOLÁ, P., 2018 - Mining, macroregional interaction and ritual practices in the South-central Andes: The first evidence for turquoise exploitation from the Late Prehispanic and Inca periods in North-western Argentina (Cueva Inca Viejo, Puna de Salta). Journal of Archaeological Science: Reports, 17: 81-92.

LYNCH, J., GIOVANNETTI, M. \& PÁEZ, M. C., 2010 - Ushnus de las provincias del sur del Tawantinsuyu. Comparación entre las estructuras de los sitios El Shincal y Hualfín Inka en el centro de Catamarca (Argentina). Revista de Arqueología Americana, 28: 49-81.

McEWAN, C., 2014 - Cognising and Marking the Andean Landscape: Radial, Concentric and Hierarchical Perspectives. In: Inca Sacred Space: Landscape, Site and Symbol in the Andes (F. Meddens, K. Willis, C. McEwan \& N. Branch, eds.): 29-47; Londres: Archetype Publications.

MEDDENS, F., 2014 - Boundaries at the Roof of the World: The Ushnu and Divisions in Political and Religious Space. In: Inca Sacred Space: Landscape, Site and Symbol in the Andes (F. Meddens, K. Willis, C. McEwan \& N. Branch, eds.): 57-70; Londres: Archetype Publications.

MONTEVERDE SOTIL, L. R., 2011 - La configuración arquitectónica de los ushnus como espacios de libaciones y ofrendas líquidas durante el Tahuantinsuyo. Bulletin de I'Institut Français d'Études Andines, 40 (1): 31-80.

MORALEJO, R. A., 2011 - Los Inkas al sur del Valle de Hualfín: organización del espacio desde una perspectiva paisajística; La Plata: Universidad Nacional de La Plata, Facultad de Ciencias Naturales y Museo. Tesis de doctorado. 
PALMA, J. R., FERNÁNDEZ DO RÍO, S. C., RUNCIO, M. A. \& CAPIZZI, L., 2006 - Control Imperial Inka en Campo Morado (Quebrada de Humahuaca, Provincia de Jujuy). Runa, 26 (1): 165-182.

PLATT, T., BOUYSSE-CASSAGNE, T. \& HARRIS, O. (eds.), 2006 - Qaraqara-Charka: Mallku, Inka y Rey en la provincia de Charcas (siglos XV-XVII). Historia antropológica de una confederación aymara, 1088 pp.; La Paz: Instituto Francés de Estudios Andinos, Plural editores, University of St. Andrews, University of London, Inter American Fundation, Fundación Cultural del Banco Central de Bolivia.

RAFFINO, R. A., 1981 - Los Inkas del Kollasuyu. Origen, naturaleza y transfiguraciones de la ocupación inka en los Andes Meridionales, 314 pp.; La Plata: Ramos Americana.

SOTO RODRÍGUEZ, C. \& SALAZAR SUTIL, D., 2016 - Mullu (Spondylus sp.) en el Complejo Minero San José del Abra (Alto Loa, Norte de Chile). Intersecciones en Antropología, 17: 129-135.

WILLIAMS, V. I., 2000 - El imperio Inka en la Provincia de Catamarca. Intersecciones en Antropología, 1: 55-78.

WILLIAMS, V., VILLEGAS, M. P., GHEGGI, M. S. \& CHAPARRO, M. G., 2005 - Hospitalidad e intercambio en los valles mesotermales del Noroeste Argentino. Boletín de Arqueología PUCP, 9: 335-372.

ZUIDEMA, R. T., 2014 - The Ushnus of Cusco and Sacred Centres in Andean Ethnography, Ethnohistory and Archaeology. In: Inca Sacred Space: Landscape, Site and Symbol in the Andes (F. Meddens, K. Willis, C. McEwan \& N. Branch, eds.): 5-28; Londres: Archetype Publications. 